Check for updates

Cite this: RSC Adv., 2017, 7, 21446

Received 22nd December 2016

Accepted 9th April 2017

DOI: 10.1039/c6ra28551a

rsc.li/rsc-advances

\section{A highly zinc-selective ratiometric fluorescent probe based on AIE luminogen functionalized coordination polymer nanoparticles $\dagger$}

\author{
Na Lin, Qin Zhang, Xin Xia, Mengyu Liang, Shihong Zhang, Liyan Zheng, (D) * Qiue Cao* \\ and Zhongtao Ding
}

Coordination polymer nanoparticles (CPNs) formed by self-assembly of metal ions (or clusters) and organic bridging ligands through coordination bonds provide a unique platform for designing multifunctional nanoparticles. In this work, we report a ratiometric fluorescent probe for $\mathrm{Zn}^{2+}$ based on CPNs, which were prepared from AIE fluorophore HDBB molecules with metal ions. The CPNs are composed of HDBB molecules with $\mathrm{Tb}^{3+}$ (named Tb-HDBB-CPNs) which displayed a matrix coordination-induced emission peak at a wavelength of $590 \mathrm{~nm}$, while CPNs formed by HDBB molecules and $\mathrm{Zn}^{2+}$ (named $\mathrm{Zn}$-HDBB-CPNs) showed a distinctive fluorescent property with a blue emission peak wavelength of $470 \mathrm{~nm}$. Based on the cation exchange process of Tb-HDBB-CPNs with $\mathrm{Zn}^{2+}$, a highly selective ratiometric fluorescent probe for the determination of $\mathrm{Zn}^{2+}$ in aqueous solution was developed with a linear range from 0.1 to $60 \mu \mathrm{M}$ and a detection limit of $50 \mathrm{nM}$. Our approach using AlE molecules as organic ligands for the construction of CPNs paves a way toward AIE functionalized materials with ratiometric fluorescence response and will find wide applications in chemical sensing.

\section{Introduction}

Coordination polymer nanoparticles, which are made from metal ions (or clusters) connected by organic ligands, have attracted growing interest because of their structural tailorability with different morphologies and physicochemical properties. $^{\mathbf{1 , 2}}$ To date, CPNs have been widely studied for their potential applications in gas storage and separation, heterogeneous catalysis, chemical sensing, biomedical imaging and drug delivery. ${ }^{3-11}$ Nevertheless, the rational design of functional coordination polymers nanoparticles is still in an premature, primary stage. ${ }^{12}$ Fluorescent CPNs have demonstrated their considerable potential in chemical sensing because of their hybrid structures that can offer tunable fluorescence and have been investigated in the determination of ion, drug and enzyme activity. ${ }^{13-16}$ However, the development of fluorescent CPNs has suffered from the problem of aggregation-caused quenching (ACQ), in which chromophores that show high fluorescence quantum yields in dilute solutions become nonfluorescent in the colloidal or solid state, where intermolecular interactions, such as $\pi$-stacking, often cause self-quenching. ${ }^{17,18}$

Key Laboratory of Medicinal Chemistry for Natural Resource (Yunnan Univeristy), Ministry of Education, School of Chemical Science and Technology, Yunnan University, Kunming, Yunnan 650091, P. R. China. E-mail: zhengliyan@ynu.edu.cn; qecao@ynu.edu.cn

$\dagger$ Electronic supplementary information (ESI) available. See DOI: $10.1039 / \mathrm{c} 6 \mathrm{ra} 28551 \mathrm{a}$
The concept of aggregation-induced emission or aggregationinduced enhanced emission (AIEE) has emerged to be a powerful and versatile strategy for the design of novel types fluorescent probes and luminescent materials to overcome ACQ problem. ${ }^{\mathbf{1 9 - 2 1}}$ In this process, the fluorogens are almost non-emissive as individual molecule because of the nonradiative decay through intramolecular motion. Such intramolecular motion can be restricted once these molecules aggregate together, resulting in strong fluorescence. A typical example is tetraphenylethene (TPE) molecule, a well-known AIE fluorogen, in which the olefin stator is surrounded by phenyl rotors and the restriction of intramolecular motion (RIM) is known to account for its strong fluorescence in colloids and in the solid state. ${ }^{22,23}$ AIE-based luminescent materials not only avoid the ACQ problem caused by aggregation but also provide new approaches based on turnon mode in the applications of chemical sensors, ${ }^{24-29}$ biological probes, ${ }^{30-33}$ and solid-state emitters. ${ }^{34,35}$

Recently, some TPE-based AIE molecules were utilized as organic ligands in the construction of metal-organic frameworks (MOFs), which is an intriguing class of inorganic-organic hybrid crystalline materials. ${ }^{36,37}$ These studies demonstrated that rigidifying AIE molecules as fluorescent links by MOFs formation efficiently and substantially tune the electronic transition energies and raise the florescent quantum yield, owing to twisted linker conformation, intramolecular hindrance, and framework rigidity. ${ }^{38}$ The applications of TPEbased MOFs with excellent luminescent behaviors are well explored as light-emitting diodes (LED), drug vehicles and 
chemical sensor. ${ }^{39-42}$ On the other hand, to the best of our knowledge, the utilization of AIE molecules as organic ligands in the amorphous CPNs has not been investigated. Different from the crystal CPNs with static structures, the amorphous CPNs exhibit a higher level of structural tailorability. Their structures can be depolymerized much faster under milder conditions. ${ }^{43}$ Moreover, the preferred turn-on responsive fluorescence when exposed to analytes was not obvious due to the almost complete restriction of intramolecular motion (RIM) in the ligands after MOFs formation..$^{39,40}$ One way to overcome this disadvantage is developing ratiometric fluorescent probes, which display two distinctly different measurable signals in the presence and absence of analyte.

Zinc is the second most abundant heavy metal ion, after iron, in the human body and plays important roles in various biological systems. It is an essential element required by all cells with various functions, for example, the control of gene transcription, metalloenzyme function and synaptic vesicles in excitatory nerve terminals. ${ }^{44}$ Thus, zinc ions are important analyte from the view of biochemistry, medicine and environment. ${ }^{45}$ Taking envisaged applications into account, especially interesting are zinc ions probes operating in ratiometric fluorimetric mode. ${ }^{46}$

Herein, we report a new type AIE-based CPNs that is prepared from AIE fluorophore HDBB molecules with metal ions. Tb-HDBB-CPNs and Zn-HDBB-CPNs emit different wavelength fluorescence in aqueous solution upon excitation with $365 \mathrm{~nm}$ light. The cation exchange process of these AIE-based CPNs were studied by fluorescence emission spectra, energydispersive X-ray (EDX) spectra, and inductively coupled plasma (ICP) measurement. A ratiometric fluorescent probe for the detection of $\mathrm{Zn}^{2+}$ was developed on the basis of cation exchange process of Tb-HDBB-CPNs with $\mathrm{Zn}^{2+}$.

\section{Experimental section}

\subsection{Chemicals and materials}

2,4-Dihydroxybenzaldehyde, 4-carboxybenzaldehyde, terbium nitrate pentahydrate, zinc nitrate pentahydrate, disodium hydrogen phosphate and sodium dihydrogen phosphate were purchased from Aladdin. 4-Formyl-3-hydroxybenzoic acid were purchased from Bide pharmatech. All other reagents were of analytical grade and used as received. Doubly distilled water was used throughout the experiments. Tris-HCl (Tris $=2$ amino-2-hydroxymethylpropane-1,3-diol) buffered solutions were prepared by titrating $0.01 \mathrm{M}$ Tris solutions with a concentrated $\mathrm{HCl}(1 \mathrm{M})$ to the required $\mathrm{pH}$ values.

\subsection{Apparatus}

X-ray powder diffraction patterns were taken using a D/max-TTR III X-ray diffractometer (Rigaku, Japan) with a scan speed of $0.1 \mathrm{~s}$ per step and a step size of $0.01^{\circ}$. The morphology and composition element of CPNs were characterized by field emission scanning electron microscopy (SEM) (JEM-2100F). The Fourier transform infrared (FT-IR) spectra were obtained on a FT-IR spectrophotometer (Thermo Nicolet 365). UV-Vis absorption was characterized using a UV/Vis/NIR spectrophotometer (Shimadzu, Japan). Fluorescence spectra were recorded on a Hitachi High-Technologies Corporation Tokyo Japan 5J20004 model F-7000 FL spectrofluorometer. Fluorescence emission lifetimes were determined on an Edinburgh Analytical Instrument (FLS900 fluorescence spectrometer) with a lightemitting diode lamp (405 nm) and analyzed by the use of a program for exponential fits. ICP-1000 system (Shimadzu, Japan) was utilized to the detection of metal ion concentration. Particle size distribution of HDBB-CPNs was recorded by a dynamic light scattering instrument (BI-90 Plus, Brookhaven Instruments Corp).

\subsection{Synthesis of HDBB, L2 and L3}

The synthesis of HDBB (4,4'-(hydrazine-1,2-diylidene bis (methanylylidene)) bis (3-hydroxybenzoic acid)), L2 and L3 were the same as our previous report. ${ }^{47}$ Typically, 4 -formyl-3hydroxybenzoic acid $(166.2 \mathrm{mg}, 1.0 \mathrm{mmol})$ was dissolved in methanol $(10 \mathrm{~mL})$. Then hydrazine hydrate $(24 \mu \mathrm{L}, 0.5 \mathrm{mmol}$; $80 \%$ ) was added and refluxed at $75^{\circ} \mathrm{C}$. After refluxing overnight, the resulting precipitate was filtered and washed with $30 \mathrm{~mL}$ methanol. After drying, HDBB was obtained as a yellow powder. The molecular structures of HDBB, L2 and L3 was verified by NMR and mass spectroscopy.

\subsection{Preparation of CPNs}

The preparation of the AIE-based CPNs was performed as reported previously. ${ }^{15}$ Briefly, Tb-HDBB-CPNs was synthesized by mixing an aqueous solution of $\mathrm{Tb}\left(\mathrm{NO}_{3}\right)_{3} \cdot 6 \mathrm{H}_{2} \mathrm{O}(26.11 \mathrm{mg}, 0.06$ $\mathrm{mmol})$ with Tris buffer $(10 \mathrm{~mL})$ containing HDBB $(3.28 \mathrm{mg}, 0.01$ mmol), adjusting the $\mathrm{pH}$ value to 9.0 to keeping HDBB molecules at dissolved state, and then stirring 2 hours at room temperature to form a yellow suspension. The resulting precipitate was then centrifuged and washed with $\mathrm{pH} 9.0$ Tris buffer solution several times. For the preparation of Zn-HDBBCPNs, the $\mathrm{Tb}\left(\mathrm{NO}_{3}\right)_{3} \cdot 6 \mathrm{H}_{2} \mathrm{O}$ was replaced by $\mathrm{Zn}\left(\mathrm{NO}_{3}\right)_{3} \cdot 6 \mathrm{H}_{2} \mathrm{O}$ $(11.36 \mathrm{mg}, 0.06 \mathrm{mmol})$ and also get a yellow suspension. The dynamic light scattering measurement has been applied to investigate the stability of these CPNs. The particle sizes of TbHDBB-CPNs were about $50 \pm 5 \mathrm{~nm}$ and $663 \pm 28 \mathrm{~nm}$ after storing one month and three months in doubly distilled water at $4{ }^{\circ} \mathrm{C}$ (Fig. S1 $\dagger$ ). Zn-HDBB-CPNs displayed similar tendency. These results indicated that the HDBB-CPNs remained stable at least one month.

\subsection{Quantification of HDBB, $\mathrm{Tb}^{3+}$, and $\mathrm{Zn}^{2+}$ in CPNs}

HDBB molecules were dissolved in $\mathrm{pH} 11.00 .1 \mathrm{M}$ phosphate buffer solution to obtain a series of HDBB solutions with concentrations ranging from 5.0 to $200.0 \mu \mathrm{M}$. Absorption spectra were recorded with a UV-Vis spectrophotometer. The standard curves were plotted from linear fitting of the absorbance at $365 \mathrm{~nm}$ as a function of HDBB concentrations. The samples of Tb-HDBB-CPNs and Zn-HDBB-CPNs were dissolved in $\mathrm{pH} 11.00 .1 \mathrm{M}$ phosphate solution. The amounts of HDBB, $\mathrm{Tb}^{3+}$ and $\mathrm{Zn}^{2+}$ in these samples was quantified using UV-Vis spectrophotometer and ICP measurement, respectively. 


\subsection{Fluorescence quantum yield}

The fluorescence quantum yield was calculated from the relation:

$$
\phi_{\mathrm{x}}=\phi_{\mathrm{s}}\left(\frac{n_{\mathrm{x}}}{n_{\mathrm{s}}}\right)^{2}\left(\frac{A_{\mathrm{s}}}{A_{\mathrm{x}}}\right)\left(\frac{F_{\mathrm{x}}}{F_{\mathrm{s}}}\right)
$$

where the subscripts $\mathrm{s}$ and $\mathrm{x}$ indicate the standard and text respectively, $\phi$ is the fluorescence quantum yield, $A$ corresponds to the absorbance of the solution, $F$ is the fluorescence intensity, and $n$ is the refractive index of the solvent. The quantum yields of Tb-HDBB-CPNs and Zn-HDBB-CPNs are $14.27 \%$ and $17.25 \%$, respectively.

\subsection{Analysis of real sample}

A water sample was collected from Green Lake (Yunnan, China). The sample was filtered through a $0.22 \mu \mathrm{m}$ membrane (Millipore) prior to be detected. One hundred microliters of the above water sample was added to $900 \mu \mathrm{L}$ of Tris buffer solution (10 mM, pH = 7.0) containing Tb-HDBB-CPNs $\left(20 \mu \mathrm{g} \mathrm{mL}^{-1}\right)$ and then analyzed using the developed detection method.

\section{Results and discussion}

\subsection{Preparation and characterization of AIE-based CPNs}

A typical route for preparing CPNs is shown as Scheme 1. The Tb-HDBB-CPNs were prepared from HDBB (L1) and terbium nitrate hexahydrate in $\mathrm{pH}$ 9.0 Tris buffer solution at room temperature by self-assembly process. The resulting yellow products were purified by centrifugation and washed several times with Tris buffer solution $(\mathrm{pH}=9.0)$. The resulting CPNs were suspended in aqueous solution and could be stored at $4{ }^{\circ} \mathrm{C}$ for three months without any changes. The control experiment showed that no CPNs were obtained in the absence of HDBB molecules or $\mathrm{Tb}^{3+}$, suggesting that both HDBB and $\mathrm{Tb}^{3+}$ are essential for the construction of CPNs. The FT-IR spectrum (Fig. 1A and B) measurement of the asprepared Tb-HDBB-CPNs confirm the coordination of the carboxylate groups to $\mathrm{Tb}^{3+}$, as evidenced by a shift in the $\mathrm{C}-\mathrm{O}$ stretching frequency to $1650 \mathrm{~cm}^{-1}$. Note that the original $\mathrm{C}-\mathrm{O}$ stretching frequency of the uncoordinated HDBB was observed at $1692 \mathrm{~cm}^{-1}$. The peak located at $1435 \mathrm{~cm}^{-1}$ also indicated the presence of deprotonated carboxylic acid bonded within the CPNs. ${ }^{48} \mathrm{Zn}-\mathrm{HDBB}-\mathrm{CPNs}$ show similar IR absorbance behaviors with the one of Tb-HDBB-CPNs,

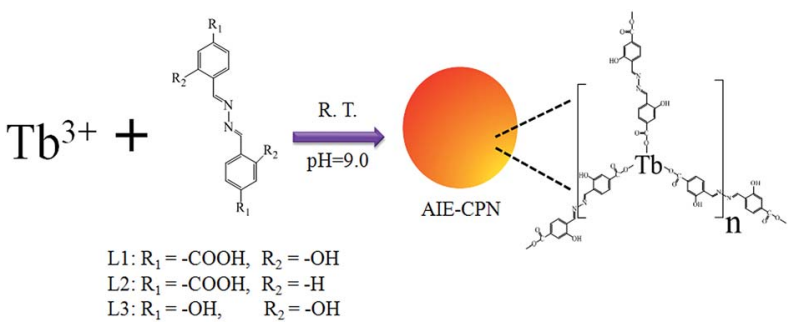

Scheme 1 Schematic representation of the preparation of AIE-based CPN.

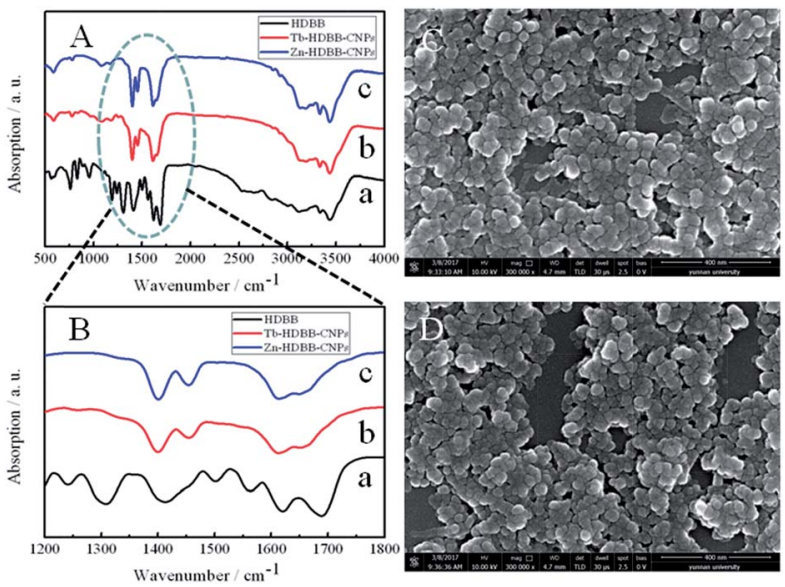

Fig. 1 (A) and (B) FTIR spectra of HDBB (a), Tb-HDBB-CPNs (b), and $\mathrm{Zn}$-HDBB-CPNs (c). FE-SEM images of Tb-HDBB-CPNs (C) and ZnHDBB-CPNs (D).

suggesting that the formation of Zn-HDBB-CPNs was underwent similar coordination process between HDBB molecule with $\mathrm{Zn}^{2+}$. To further validate the effect of carboxylate group on the formation of these CPNs, two salicylaldehyde hydrazine derivatives $(\mathrm{L} 2, \mathrm{~L} 3)$ were designed as organic links in the preparation of CPNs (Scheme 1). The experiment results demonstrate that CPNs can be formed in the presence of L2 with $\mathrm{Tb}^{3+}$, however, there were no CPNs obtained under similar condition with the replacement of L1 by L3 due to the lack of carboxylate group in para-position of L3. From these results, we can conclude that carboxylate group is essential for the formation of these CPNs.

The SEM images of Tb-HDBB-CPNs and Zn-HDBB-CPNs show typically spherical morphology with relatively uniform size of $50 \mathrm{~nm}$ and $55 \mathrm{~nm}$, respectively (Fig. 1C and D). The chemical composition of these CPNs was determined by energydispersive X-ray (EDX) analysis during SEM measurement (Fig. S2 and S3 $\uparrow$ ), which demonstrates the presence of $\mathrm{Tb}$ and $\mathrm{Zn}$ element, further indicating that $\mathrm{Tb}$ and $\mathrm{Zn}$ ions were involved in the construction of Tb-HDBB-CPNs and Zn-HDBBCPNs, respectively. The lack of diffraction peaks in powder $\mathrm{X}$ ray diffraction (PXRD) measurement of these CPNs reveals the amorphous structure of these nanoparticles (Fig. S4†).

To further elucidate the structure of these CPNs, the amounts of HDBB molecules and metal ions in these CPNs were quantified using UV-Vis spectra and ICP measurement (Fig. S5-S7 $\dagger$ ), respectively. The ratios of metal ions to HDBB molecules in these CPNs are about $1: 1.5$ for Tb-HDBB-CPNs and $1: 1$ for Zn-HDBB-CPNs. As a result, the HDBB molecules were in the negative state in the preparation condition $(\mathrm{pH}=9.0)$ of these AIE-based CPNs, and the cations electrostatically attached to the HDBB molecule forming these CPNs. Therefore, the formation of these AIE-based coordination polymers mediated by strong metal-ligand bonding is dominant in the self-assembly process. According to the above results, the structure of Tb-HDBB-CPNs can be proposed and shown in Scheme 1. 


\subsection{Photochemical properties of AIE-based CPNs}

To investigate photochemical properties of these AIE-based CPNs, the fluorescence and absorption spectra were measured. The absorption spectra of these AIE-based CPNs exhibit two peaks at $310 \mathrm{~nm}$ and $365 \mathrm{~nm}$ (Fig. 2B), which are the same to that of $\mathrm{HDBB},{ }^{47}$ and the absorption intensities are slightly increasing. These absorptions can be ascribed to the $\mathrm{p}-$ $\mathrm{p}^{*}$ and $\mathrm{n}-\mathrm{p} *$ transitions of the entire molecule.

The fluorescence spectra shows that the maxima emission and excitation of Tb-HDBB-CPNs are at $590 \mathrm{~nm}$ and $365 \mathrm{~nm}$ (Fig. 2A), which are similar to the precursor HDBB. The fluorescence quantum yield of Tb-HDBB-CPNs is $14.27 \%$ in aqueous solution, which is about three times larger than the one of HDBB $(5.32 \%)$. The fluorescence enhancement of TbHDBB-CPNs can be attributed to the immobilization of the HDBB linker as it is strongly coordinated to $\mathrm{Tb}^{3+}$. The formation of rigid CPNs via coordination of HDBB to $\mathrm{Tb}^{3+}$ prevents the torsional relaxation, thus the RIM process is activated. The fluorescence lifetime of Tb-HDBB-CPNs (2.92 ns) is longer than the one of HDBB molecules (1.97 ns) (Fig. S8 $\dagger$ ). After coordinating with $\mathrm{Tb}^{3+}$, the radiative rate constant $\left(k_{\mathrm{r}}\right)$ of the HDBB increased from $27.0 \times 10^{6} \mathrm{~s}^{-1}$ to $48.86 \times 10^{6} \mathrm{~s}^{-1}$, but the nonradiative rate constant $\left(k_{\mathrm{nr}}\right)$ decreased from $4.80 \times 10^{8} \mathrm{~s}^{-1}$ to $2.94 \times 10^{8} \mathrm{~s}^{-1}$ (Table S1 $\dagger$ ).

These results are similar with previous reports, the lifetime and fluorescence quantum yield of many AIE molecules usually increase after aggregation owing to the reducing non-radiative processes and activating the radiative channel. ${ }^{19}$ Consequently, the formation of CPNs prevents the HDBB excited state distortion, hence the exciton immediately released the energy via fluorescence and results in a higher fluorescence quantum yield and longer lifetime.

Unlike Tb-HDBB-CPNs emit yellow fluorescence measured at $590 \mathrm{~nm}$, the Zn-HDBB-CPNs emit blue lights observed at $470 \mathrm{~nm}$, which is almost $120 \mathrm{~nm}$ blue-shifted (Fig. 2A). The maximum excitation wavelength of $\mathrm{Zn}-\mathrm{HDBB}-\mathrm{CPNs}$ is retained at $365 \mathrm{~nm}$, which is consistent with its absorption spectrum. The lifetime and fluorescence quantum yield are $17.25 \%$ and $1.01 \mathrm{~ns}$ (Fig. S9†). The radiative rate constant and non-radiative rate constant of $\mathrm{Zn}$-HDBB-CPNs were $172.5 \times 10^{6} \mathrm{~s}^{-1}$ and 8.19 $\times 10 \mathrm{~s}^{-1}$, respectively. This distinct emission can be ascribed to the inhibition of photo-induced electron transfer process after
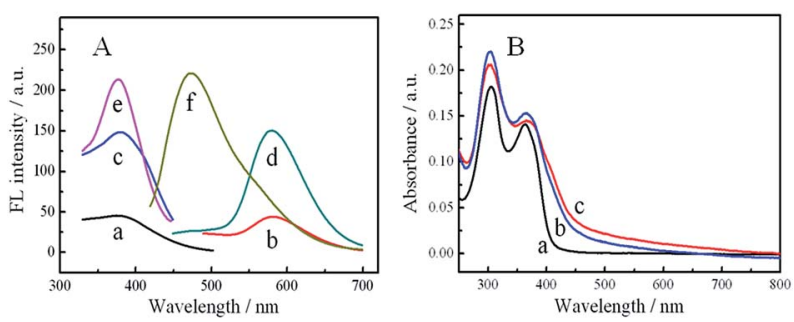

Fig. 2 (A) Excitation and emission spectra of $0.1 \mathrm{mM} \mathrm{HDBB}$ ( $a$ and b), $0.035 \mathrm{mg} \mathrm{mL}^{-1} \mathrm{~Tb}-\mathrm{HDBB}-\mathrm{CPNs}$ (c and d), and $0.035 \mathrm{mg} \mathrm{mL}^{-1} \mathrm{Zn}$ HDBB-CPNs (e and f). The emission spectra were recorded at $365 \mathrm{~nm}$ excitation wavelength. (B) UV-Vis spectra of $0.1 \mathrm{mM}$ HDBB (a), 0.035 $\mathrm{mg} \mathrm{mL}^{-1} \mathrm{~Tb}-\mathrm{HDBB}-\mathrm{CPNs}$ (b), and $0.035 \mathrm{mg} \mathrm{mL}^{-1} \mathrm{Zn}-\mathrm{HDBB}-\mathrm{CPNs}$ (c).
HDBB coordinating with $\mathrm{Zn}(\mathrm{II})$; two nitrogens on hydrazone moiety in HDBB molecule as well as phenolic oxygen may participate in binding to $\mathrm{Zn}^{2+} \cdot{ }^{49,50}$

\subsection{Sensing mechanism of ratiometric fluorescent probe for $\mathrm{Zn}^{2+}$}

Cation exchange in CPNs and MOFs has become a powerful method tuning their composition, functionality and porosity. ${ }^{6,51,52}$ We hypothesized that cation exchange process also can occur in our AIE-based CPNs. In a typical reaction, we prepared a mixture of Tb-HDBB-CPNs and $0.15 \mathrm{mM} \mathrm{Zn}^{2+}$ in $\mathrm{pH}$ 7.0 Tris buffer solution under ambient conditions. After $3 \mathrm{~h}$ of cation exchange, the resulted CPNs were separated from the mixture by centrifugation. Fluorescence emission spectra, SEM, EDX spectra and ICP spectra were measured before and after cation exchange reaction of the CPNs sample. As shown in Fig. 3A, fluorescence emission centered at $470 \mathrm{~nm}$ increased remarkably with a decrease of fluorescence emission at $590 \mathrm{~nm}$ under the excitation wavelength at $365 \mathrm{~nm}$. The dual emission spectrum of the resulting particles reveals successful cation exchange of Tb-HDBB-CPNs by $\mathrm{Zn}^{2+}$, consequently, blocking of photo-induced electron transfer process of HDBB molecules. Furthermore, the morphology of TbHDBB-CPNs after $\mathrm{Zn}^{2+}$ exchange is not much different from the one of before exchange (Fig. S10 $\dagger$ ). A relating EDX spectrum of the Tb-HDBB-CPNs measured after the cation exchange shows the expected $\mathrm{Zn}$ signal, meanwhile, Tb signal is also can be observed in the EDX spectrum (Fig. S11†). Finally, Tb signal observed in ICP spectrum of Tb-HDBB-CPNs supernatant after cation exchange is much larger than the one of control experiment without adding $\mathrm{Zn}^{2+}$, indicating that partly cation exchange of Tb-HDBB-CPNs by $\mathrm{Zn}^{2+}$. These experiments provided direct evidences of cation exchange in Tb-HDBB-CPNs with $\mathrm{Zn}^{2+}$. Thus, the cation exchange process of Tb-HDBB-CPNs is demonstrated as in Fig. 3B. Briefly, upon the addition of $\mathrm{Zn}^{2+}$, some carboxylic group on the surface of Tb-HDBB-CPNs bind to $\mathrm{Zn}^{2+}$ resulting in the formation $\mathrm{Zn}-\mathrm{Tb}$ HDBB-CPNs with dual emission fluorescent behavior. As a result, our strategy not only provides a facile route to introduce desired functionality into CPNs but also promotes the potential of CPNs for application, such as ratiometric fluorescence detection.

\subsection{Optimization for $\mathrm{Zn}^{2+}$ ion detection}

As is demonstrated above, the successful cation exchange process of $\mathrm{Tb}$-HDBB-CPNs by $\mathrm{Zn}^{2+}$, inducing the increasing

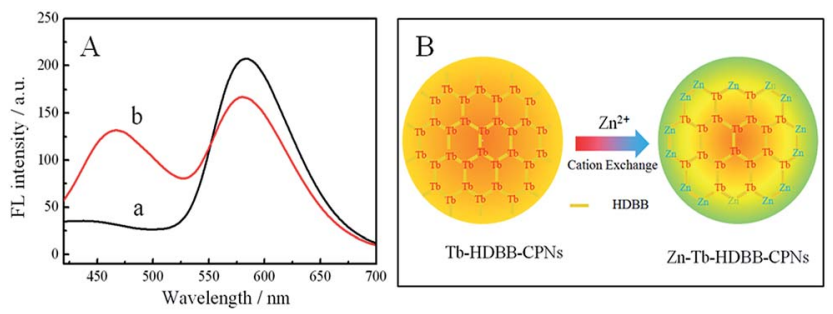

Fig. 3 (A) Fluorescence emission spectra of $0.035 \mathrm{mg} \mathrm{mL}^{-1} \mathrm{~Tb}-$ HDBB-CPNs in the absence (curve a) and presence of $0.15 \mathrm{mM} \mathrm{Zn^{2+ }}$ (curve b). (B) Schematic representation of the cation exchange process of Tb-HDBB-CPNs with $\mathrm{Zn}^{2+}$. 
fluorescence at $470 \mathrm{~nm}$ and the decreasing emission at $590 \mathrm{~nm}$, prompted us to apply this AIE-based CPNs for the detection of $\mathrm{Zn}^{2+}$ in aqueous solution. We can image that the ratios of $I_{470}$ to $I_{590}$ (i.e., $I_{470} / I_{590}$ ) would be closely associated with the degree of the exchange of the Tb-HDBB-CPNs caused by $\mathrm{Zn}^{2+}$, as a consequence, which could be used for the ratiometric fluorescence assay of $\mathrm{Zn}^{2+}$.

Fig. 4A depicts the typical time-dependent fluorescent intensity at $470 \mathrm{~nm}$ of the Tris buffer containing Tb-HDBB-CPNs after the addition of $\mathrm{Zn}^{2+}$ into the buffer. Upon the addition of $\mathrm{Zn}^{2+}$ the fluorescent spectrum of the Tb-HDBB-CPNs was recorded immediately and every on minute consecutively at room temperature. After adding $\mathrm{Zn}^{2+}$, the fluorescent intensity at $470 \mathrm{~nm}$ of $\mathrm{Tb}-\mathrm{HDBB}-\mathrm{CPNs}$ was enhanced significantly and reached a plateau after $2 \mathrm{~min}$. The fast fluorescence response can be ascribed to the unique amorphous structure of $\mathrm{Tb}$ HDBB-CPNs.

We next examined the effects of other metal ions on the fluorescence intensity of Tb-HDBB-CPNs under identical conditions. As shown in Fig. 4B, only the addition of $\mathrm{Zn}^{2+}$ resulted in a significant fluorescence enhancement at $470 \mathrm{~nm}$; no remarkable changes in the fluorescence of Tb-HDBB-CPNs were observed upon the addition of other metal ions, including $\mathrm{Co}^{2+}, \mathrm{Mg}^{2+}, \mathrm{K}^{+}, \mathrm{Fe}^{3+}, \mathrm{Ni}^{2+}, \mathrm{Ca}^{2+}, \mathrm{Cd}^{2+}, \mathrm{Pb}^{2+}, \mathrm{Hg}^{2+}$, and $\mathrm{Cu}^{2+}$. The high selectivity of Tb-HDBB-CPNs to $\mathrm{Zn}^{2+}$ is attributed to the special fluorescent behavior of the coordination compound of $\mathrm{Zn}^{2+}$ with HDBB.

The fluorescence response of Tb-HDBB-CPNs to $\mathrm{Zn}^{2+}$ in different concentrations is shown as Fig. 4C. Corresponding to the decreased fluorescence at $590 \mathrm{~nm}$, the fluorescence at
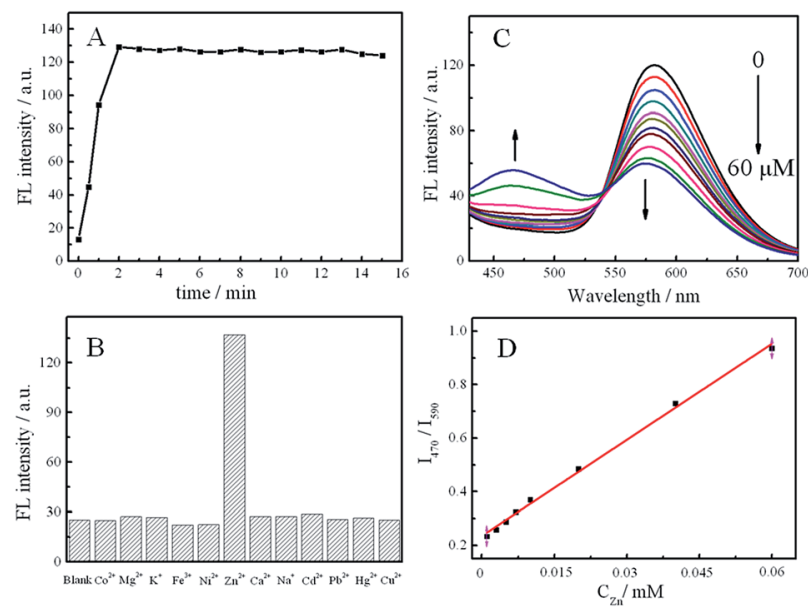

Fig. 4 (A) Time-dependent fluorescence at $470 \mathrm{~nm}$ response of the $0.02 \mathrm{mg} \mathrm{mL}^{-1} \mathrm{~Tb}-\mathrm{HDBB}-\mathrm{CPNs}$ to $0.1 \mathrm{mM} \mathrm{Zn}^{2+}$ in $\mathrm{pH} 7.0$ Tri buffer solution. (B) The selectivity of Tb-HDBB-CPNs based sensor for $\mathrm{Zn}^{2+}$ to other cations in $\mathrm{pH}$ 7.0 Tri buffer solution. The concentrations of $\mathrm{Tb}-\mathrm{HDBB}-\mathrm{CPNs}, \mathrm{Zn}^{2+}$ and other cations were $0.02 \mathrm{mg} \mathrm{mL}^{-1}, 0.15 \mathrm{mM}$ and $0.25 \mathrm{mM}$, respectively. (C) Fluorescence emission spectra of TbHDBB-CPNs probe exposed to various concentrations of $\mathrm{Zn}^{2+}$. These spectra were measured in $\mathrm{pH}$ 7.0 Tri buffer solution. The concentration of Tb-HDBB-CPNs was $0.02 \mathrm{mg} \mathrm{mL}^{-1}$. The concentration of $\mathrm{Zn}^{2+}$ were $0,0.1,0.5,1.0,3.0,5.0,7.0,10,20,40,60 \mu \mathrm{M}$. (D) The linear plot of the value of $I_{470} / I_{590}$ versus the concentration of $\mathrm{Zn}^{2+}$.
$470 \mathrm{~nm}$ was enhanced gradually with the increase of $\mathrm{Zn}^{2+}$ concentrations from 0 to $60 \mu \mathrm{M}$. There is a linear ratio fluorescence response to $\mathrm{Zn}^{2+}$ in the concentration range 0.1-60 $\mu \mathrm{M}$. The detection limit is $50 \mathrm{nM}$ on the basis of a signal-tonoise ratio of $3: 1$. This detection limit satisfies the maximum permitted level of $5 \mathrm{mg} \mathrm{L}^{-1} \mathrm{Zn}^{2+}$ in drinking water regulated by the U.S. Environmental Protection Agency (EPA). ${ }^{45}$

\subsection{Application in water samples}

It is very important to monitor the level of $\mathrm{Zn}^{2+}$ in water samples, because $\mathrm{Zn}^{2+}$ in human bodies tend to bio-accumulate and damage central nervous system, blood composition, lungs, kidneys and liver. The practicability of Tb-HDBB-CPNs fluorescent nanoprobes was assessed by applying it to the real analysis for $\mathrm{Zn}^{2+}$ in water sample of the Green Lake, which is a famous scenic spot of Yunnan Province in China. An average value of $1.48 \pm 0.18 \mu \mathrm{M} \mathrm{Zn}^{2+}$ was found for $n=4$ determinations in the Green Lake water sample using this new approach with good recovery $(99.15 \%)$, after the subtraction of the interception from that of the standard calibration curve (Fig. 4D), which is consistent with the result obtained by ICP spectroscopic methods, $1.34 \pm 0.08 \mu \mathrm{M}$. This result suggests that our ratiometric fluorescence nanoprobe can be used for $\mathrm{Zn}^{2+}$ determination in water samples.

\section{Conclusions}

In summary, AIE-based CPNs have been developed by using AIE molecules as bridging ligands. Tb-HDBB-CPNs were further employed as ratiometric fluorescence probe to direct detection of $\mathrm{Zn}^{2+}$ in aqueous solution based on cation exchange effect. The ratio fluorescence of $\mathrm{Zn}$-Tb-HDBB-CPNs was enhanced linearly with the $\mathrm{Zn}^{2+}$ concentration from $100 \mathrm{nM}$ to $60 \mu \mathrm{M}$. The detection limit for $\mathrm{Zn}^{2+}$ in aqueous solution is $50 \mathrm{nM}$. The ratiometric fluorescent probe based on Tb-HDBB-CNPs exhibits the advantages of direct and fast detection procedure, excellent stability and selectivity. The proposed strategy might provide a new platform for the design and application of fluorescent probes based on AIE functionalized coordination polymers.

\section{Acknowledgements}

This work was financially supported by the National Natural Science Foundation of China (NSFC, 21405135, 21465025), the Project sponsored by SRF for Yunnan university (2013CK006) and Cultivation Program for Key Young Teachers of Yunnan University (XT412003).

\section{Notes and references}

1 M. Oh and C. A. Mirkin, Nature, 2005, 438, 651-654.

2 Y. Liu, F. Boey, L. L. Lao, H. Zhang, X. Liu and Q. Zhang, Chem.-Asian J., 2011, 6, 1004-1006.

3 I. Imaz, M. Rubio-Martínez, L. García-Fernández, F. García, D. Ruiz-Molina, J. Hernando, V. Puntes and D. Maspoch, Chem. Commun., 2010, 46, 4737-4739. 
4 C. M. Hongliang Tan, Q. Li, L. Wang, F. Xu, S. Chen and A. Song, Analyst, 2014, 139, 5516-5522.

5 K. E. deKrafft, Z. Xie, G. Cao, S. Tran, L. Ma, O. Z. Zhou and W. Lin, Angew. Chem., Int. Ed., 2009, 48, 9901-9904.

6 M. Oh and C. A. Mirkin, Angew. Chem., 2006, 118, 5618-5620.

7 N. Lin, J. Li, Z. Lu, L. Bian, L. Zheng, Q. Cao and Z. Ding, Nanoscale, 2015, 7, 4971-4977.

8 L. Xing, Y. Cao and S. Che, Chem. Commun., 2012, 48, 59955997.

9 W. J. Rieter, K. M. Pott, K. M. Taylor and W. Lin, J. Am. Chem. Soc., 2008, 130, 11584-11585.

10 Y. Liao, L. He, J. Huang, J. Zhang, L. Zhuang, H. Shen and C.-Y. Su, ACS Appl. Mater. Interfaces, 2010, 2, 2333-2338.

11 R. C. Huxford, K. E. Dekrafft, W. S. Boyle, D. Liu and W. Lin, Chem. Sci., 2012, 3, 198-204.

12 F. Pu, E. Ju, J. Ren and X. Qu, Adv. Mater., 2014, 26, 11111117.

13 H. Tan, B. Liu and Y. Chen, ACS Nano, 2012, 6, 10505-10511. 14 H. Tan, L. Zhang, C. Ma, Y. Song, F. Xu, S. Chen and L. Wang, ACS Appl. Mater. Interfaces, 2013, 5, 11791-11796.

15 J. Deng, P. Yu, Y. Wang and L. Mao, Anal. Chem., 2015, 87, 3080-3086.

16 R. Nishiyabu, N. Hashimoto, T. Cho, K. Watanabe, T. Yasunaga, A. Endo, K. Kaneko, T. Niidome, M. Murata and C. Adachi, J. Am. Chem. Soc., 2009, 131, 2151-2158.

17 K. Mizusawa, Y. Takaoka and I. Hamachi, J. Am. Chem. Soc., 2012, 134, 13386-13395.

18 K. Mizusawa, Y. Ishida, Y. Takaoka, M. Miyagawa, S. Tsukiji and I. Hamachi, J. Am. Chem. Soc., 2010, 132, 7291-7293.

19 Y. Hong, J. W. Lam and B. Z. Tang, Chem. Commun., 2009, 4332-4353.

20 J. Mei, Y. Hong, J. W. Lam, A. Qin, Y. Tang and B. Z. Tang, Adv. Mater., 2014, 26, 5429-5479.

21 Z. Luo, X. Yuan, Y. Yu, Q. Zhang, D. T. Leong, J. Y. Lee and J. Xie, J. Am. Chem. Soc., 2012, 134, 16662-16670.

22 R. Hu, N. L. Leung and B. Z. Tang, Chem. Soc. Rev., 2014, 43, 4494-4562.

23 N. B. Shustova, T.-C. Ong, A. F. Cozzolino, V. K. Michaelis, R. G. Griffin and M. Dincă, J. Am. Chem. Soc., 2012, 134, 15061-15070.

24 V. Vij, V. Bhalla and M. Kumar, ACS Appl. Mater. Interfaces, 2013, 5, 5373-5380.

25 Y. Liu, C. Deng, L. Tang, A. Qin, R. Hu, J. Z. Sun and B. Z. Tang, J. Am. Chem. Soc., 2010, 133, 660-663.

26 Y. Zhang, D. Li, Y. Li and J. Yu, Chem. Sci., 2014, 5, 27102716.

27 L. Zhang, N. He and C. Lu, Anal. Chem., 2015, 87, 1351-1357.

28 J. Chen, Y. Wang, W. Li, H. Zhou, Y. Li and C. Yu, Anal. Chem., 2014, 86, 9866-9872.

29 D. G. Khandare, H. Joshi, M. Banerjee, M. S. Majik and A. Chatterjee, Anal. Chem., 2015, 87, 10871-10877.
30 R. T. Kwok, C. W. Leung, J. W. Lam and B. Z. Tang, Chem. Soc. Rev., 2015, 44, 4228-4238.

31 J. Liang, B. Z. Tang and B. Liu, Chem. Soc. Rev., 2015, 44, 2798-2811.

32 D. Ding, K. Li, B. Liu and B. Z. Tang, Acc. Chem. Res., 2013, 46, 2441-2453.

33 X. Zhang, X. Zhang, S. Wang, M. Liu, Y. Zhang, L. Tao and Y. Wei, ACS Appl. Mater. Interfaces, 2013, 5, 19431947.

34 L. Chen, Y. Jiang, H. Nie, R. Hu, H. S. Kwok, F. Huang, A. Qin, Z. Zhao and B. Z. Tang, ACS Appl. Mater. Interfaces, 2014, 6, 17215-17225.

35 D. Li, Y. Zhang, Z. Fan, J. Chen and J. Yu, Chem. Sci., 2015, 6, 6097-6101.

36 N. B. Shustova, B. D. McCarthy and M. Dinca, J. Am. Chem. Soc., 2011, 133, 20126-20129.

38 Z. Wei, Z.-Y. Gu, R. K. Arvapally, Y.-P. Chen, R. N. McDougald Jr, J. F. Ivy, A. A. Yakovenko, D. Feng, M. A. Omary and H.-C. Zhou, J. Am. Chem. Soc., 2014, 136, 8269-8276.

37 Q. Zhang, J. Su, D. Feng, Z. Wei, X. Zou and H.-C. Zhou, J. Am. Chem. Soc., 2015, 137, 10064-10067.

39 Z. Hu, W. P. Lustig, J. Zhang, C. Zheng, H. Wang, S. J. Teat, Q. Gong, N. D. Rudd and J. Li, J. Am. Chem. Soc., 2015, 1620916215.

40 X.-G. Liu, H. Wang, B. Chen, Y. Zou, Z.-G. Gu, Z. Zhao and L. Shen, Chem. Commun., 2015, 51, 1677-1680.

41 L. Wang, W. Wang and Z. Xie, J. Mater. Chem. B, 2016, 4, 4263-4266.

42 N. B. Shustova, A. F. Cozzolino, S. Reineke, M. Baldo and M. Dincă, J. Am. Chem. Soc., 2013, 135, 13326-13329.

43 A. M. Spokoyny, D. Kim, A. Sumrein and C. A. Mirkin, Chem. Soc. Rev., 2009, 38, 1218-1227.

44 A. Takeda, M. Nakamura, H. Fujii and H. Tamano, Metallomics, 2013, 5, 417-423.

45 P. Xu, S. Huang, Z. Wang and G. Lagos, Sci. Total Environ., 2006, 362, 50-55.

46 J. Du, M. Hu, J. Fan and X. Peng, Chem. Soc. Rev., 2012, 41, 4511-4535.

47 N. Lin, X. Chen, S. Yan, H. Wang, Z. Lu, X. Xia, M. Liang, Y.-L. Wu, L. Zheng and Q. Cao, RSC Adv., 2016, 6, 2541625419.

48 C. McKinstry, E. J. Cussen, A. J. Fletcher, S. V. Patwardhan and J. Sefcik, Cryst. Growth Des., 2013, 13, 5481-5486.

49 X. Cao, X. Zeng, L. Mu, Y. Chen, R. Wang, Y. Zhang, J. Zhang and G. Wei, Sens. Actuators, B, 2013, 177, 493-499.

50 D. Xie, Z. Ran, Z. Jin, X. Zhang and D. An, Dyes Pigm., 2013, 2013(96), 495-499.

51 M. H. Lee, J. S. Kim and J. L. Sessler, Chem. Soc. Rev., 2015, 44, 4185-4191.

52 Z. Wang and S. M. Cohen, Chem. Soc. Rev., 2009, 38, 13151329. 